\title{
ÖWAV-Seminar „Kanäle, Rohre, Leitungen“ ein voller Erfolg
}

\section{Peter Sander}

Online publiziert: 4. Juni 2013

(C) Springer-Verlag Wien 2013

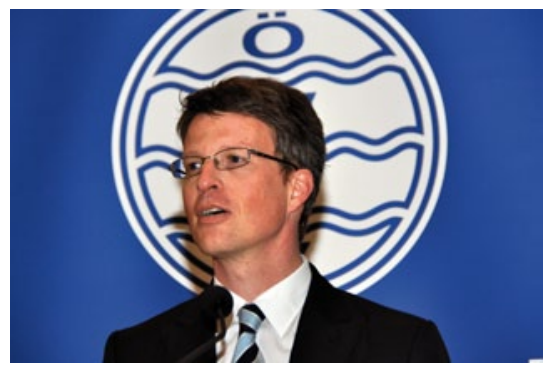

RA Mag. Martin Niederhuber bei seinem Vortrag. (Foto: ÖWAV)

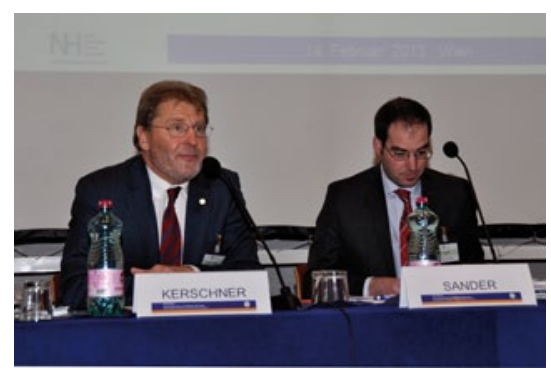

(v.I.) Univ.-Prof. Dr. Ferdinand Kerschner und RA Dr. Peter Sander, LL.M., MBA

Rund 100 SeminarteilnehmerInnen durften sich am 14. Februar des heurigen Jahres über ein abwechslungsreiches Potpourri unterschiedlichster Einblicke in das Wege- und Leitungsrecht freuen. Erstmals organisierte der Österreichische Wasser- und Abfallwirtschaftsverband (ÖWAV) gemeinsam mit der auf Infrastruktur- und Industrieanlagenrecht spezialisierten Anwaltskanzlei Niederhuber \& Partner(NHP) eine Spezialfortbildungsveranstaltung zwischen privatrechtlichen Verträgen, Zustimmungen, Erklärungen und grundbuchsrechtlichen Spezialfragen einerseits und Anlagengenehmigungsverfahren andererseits.

Wie insbesondere der Linzer Univ.Prof. Dr. Ferdinand Kerschner in seinen

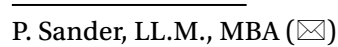

Niederhuber \& Partner Rechtsanwälte

GmbH, Wollzeile 24/12, 1010 Wien,

Österreich

E-Mail: Peter.Sander@nhp.eu einleitenden Worten trefflich bemerkte, wird das Wege- und Leitungsrecht im Allgemeinen und das Energiewegerecht im Besonderen in Zukunft wohl eine noch größere Rolle als bereits bisher spielen. Dieser Meinung waren offensichtlich auch die vielen PraktikerInnen, die insbesondere die Diskussionsblöcke mit den Referenten durch zahlreiche und oft äußerst verzwickte Probleme ansprechende Fragestellungen bereicherten.

In einem ersten Block gelang es Dr. Matthias Neubauer, dem Leiter der Sektion IV/4 Versorgungssicherheit und Energiewegerecht, vorzüglich, den Boden der Rechtsgrundlagen aufzubereiten. Er spannte dabei einen weiten Bogen über die unterschiedlichsten Materiengesetze, ohne aber die verfassungsrechtlichen Grundlagen außer Acht zu lassen. Seine Conclusio war jedoch trotz der recht komplexen Materie insofern erfreulich, als er - eine profunde Aufbereitung der Unterlagen vorausgesetzt - den SeminarteilnehmerInnen den Schrecken vor überlangen und hochkomplexen Verfahren zur Rechteeinräumung nehmen konnte.

In einem weiteren Referat wies Mag. Gernot Wallner von der NÖ Wasserrechtsbehörde geschickt einerseits auf Fallstricke bei der Verbindung von $\mathrm{Zu}$ stimmungserklärungen (zu bestimmten Projekten) und den jeweiligen Projektgenehmigungsverfahren am Beispiel des Wasserrechts hin und gab gleichzeitig praktische Tipps für die Optimierung. Den Abschluss des ersten Vortragsblocks bildete ein Beitrag von Rechtsanwalt Mag. Paul Reichel (NHP), der anschaulich aufzeigte, dass auch Jahre und Jahrzehnte nach der Errichtung von Leitungsanlagen Risiken durch gutgläubigen lastenfreien Eigentumserwerb und Ersitzung drohen. Zusammenfassend kann seinem Beitrag jedenfalls der Warnhinweis für LeitungsanlagenbetreiberInnen gleich welcher Art entnommen werden, möglichst regelmäßig den Stand der (insbesondere grundbücherlichen) Sicherstellung der jeweiligen Wege- und Leitungsrechte zu überprüfen.
Nach einer Kaffeepause folgten im zweiten eher praxisorientierten Vortragsblock drei nicht minder spannende Themen: Rechtsanwalt Dr. Peter Sander (NHP) erarbeitete gemeinsam mit den SeminarteilnehmerInnen eine Art Vertragsmuster für einen Optionsvertrag zur Absicherung der bei Grundeinlöseverhandlungen erzielten Zwischen- und Endergebnisse. Mit der Erstellung eines Vertragsmusters gleichsam in Echtzeit während des Vortrages beschritt das Veranstalterteam rund um den ÖWAV dabei Neuland. Insbesondere die angeregte Diskussion schon während des Vortrages von Dr. Sander zeigte aber, dass man nicht auf das falsche Pferd gesetzt hatte.

Mit viel Spannung wurde dann der Vortrag von Otto Lapuch (Salzburg AG) erwartet, der gleichsam „aus dem Nähkästchen“ eines Grundeinlösers plauderte und überzeugend die verschiedenen Strategien darstellte, wie man erfolgreich zu Unterschriften und Zustimmungen der jeweilig betroffenen GrundeigentümerInnen kommt. Abschließend beleuchtete Univ.-Ass. Dr. Erich Pürgy, wie ein Enteignungsverfahren richtig durchführt wird - nur für den Fall, dass sämtliche Bemühungen zur Grundeinlöse zuvor gescheitert wären. Dr. Erich Pürgy verstand es dabei auch geschickt, den SeminarteilnehmerInnen die durchwegs komplexen verfassungsrechtlichen Hintergründe praxisnah zu erläutern.

Insgesamt darf resümiert werden, dass das vom ÖWAV angebotene Seminar zum Wege- und Leitungsrecht durchwegs den Nerv der Zeit getroffen hat. Dass die Thematik eine brisante ist, hat nicht zuletzt die Zahl der TeilnehmerInnen bewiesen. Vor diesem Hintergrund wäre es wünschenswert, dass der ÖWAV Veranstaltungen wie diese auf einer regelmäßigen Basis zum Update der jeweils neuesten Entwicklungen gleichsam fix in sein Programm aufnimmt. 